\title{
Systematic study of innovative hygroscopic and non-hygroscopic crystals with SiPM array readout
}

\author{
M. Bonesini ${ }^{* 1,2}$ R.Bertoni $^{2}$, T. Cervi $i^{3,4}$, M. Clemenza $^{1,2}$, A. de Bari ${ }^{3,4}$, R. Mazza ${ }^{2}$, A. \\ Menegolli $^{3,4}$, M.C. Prata ${ }^{4}$, M. Rossella ${ }^{4}$, \\ ${ }^{1}$ Dipartimento di Fisica G. Occhialini, Universitá di Milano Bicocca \\ ${ }^{2}$ Sezione INFN Milano Bicocca \\ ${ }^{3}$ Dipartimento di Fisica, Universitá di Pavia \\ ${ }^{4}$ Sezione INFN Pavia
}

Different crystals with SiPM array readout were tested in laboratory. Hygroscopic crystals, such as $\mathrm{LaBr}_{3}: \mathrm{Ce}$ and $\mathrm{CeBr}_{3}$, and innovative non-hygroscopic crystals, such as PrLuAg and CeCAAG were studied. $4 \times 4 \mathrm{SiPM}$ arrays from different manufacturers were used as photodetectors. Best results were obtained with $\mathrm{LaBr}_{3}$ :Ce crystals read by Hamamatsu S13361 TSV arrays. For $\mathrm{LaBr}_{3}: \mathrm{Ce}$ crystals, due to their high photon yield, it was possible to implement a simple readout scheme based on CAEN V1730 FADC, without any amplification. Energy resolutions (FWHM) better than $4 \%$ were obtained at the $\mathrm{Cs}^{137}$ peak, with a detector linearity better than $2 \%$.

The European Physical Society Conference on High Energy Physics

5-12 July

Venice, Italy

\footnotetext{
* Speaker.

†E-mail: maurizio.bonesini@mib.infn.it
} 


\section{Introduction}

Compact X-rays detectors based on crystals with SiPM array readout may find application in many fields such as TOF PET imaging [1], fundamental physics such as the FAMU measurement at Riken-RAL of the Zemach proton radius [2] and homeland security. For our aims it is essential to detect low-energy X-rays in the range 100-700 KeV. PrLuAG [3] and CeCAAG [4] crystals with respect to more conventional $\mathrm{LaBr}_{3}: \mathrm{Ce}, \mathrm{CeBr}_{3}[5]$ and $\mathrm{NaI}(\mathrm{Tl})$ crystals, have the advantage to be non hygroscopic and thus do not need encapsulation. Their main properties are shown in table 1.

Table 1: Main characteristics of the crystals under test. Typical energy resolutions (FWHM in \%), from published data, are measured at the $C s^{137}$ peak with a PMT readout.

\begin{tabular}{|l|c|c|c|c|c|}
\hline Scintillators & PrLuAG & Ce:GAGG & Ce: $\mathrm{LaBr}_{3}$ & $\mathrm{CeBr}_{3}$ & $\mathrm{NaI}(\mathrm{Tl})$ \\
\hline Density $\left(\mathrm{g} / \mathrm{cm}^{3}\right)$ & 6.73 & 6.63 & 5.08 & 5.18 & 3.67 \\
Light yield $(\gamma / \mathrm{MeV})$ & 22,000 & 57,000 & 75,000 & 47000 & 38000 \\
Decay time $(\mathrm{ns})$ & 20 & $88(91 \%)$ & 30 & 25 & 250 \\
& & $258(9 \%)$ & & & \\
Peak emission $(\mathrm{nm})$ & 310 & 520 & 375 & 370 & 415 \\
Energy resolution & 4.3 & 5.3 & 2.6 & 4.0 & 7.0 \\
Hygroscopicity & no & no & yes & yes & yes \\
\hline
\end{tabular}

As one isotope $\left(L u^{176}\right)$ of naturally occurring lutetium is unstable ${ }^{1}$ an intrinsic activity for PrLuAg crystals may be expected. The same is true for $\mathrm{Ce}: \mathrm{LaBr}_{3}$ crystals, due to the presence of $L a^{138}$, that emits conversion electrons and $\beta$ particles with energy up to $1.7 \mathrm{MeV}$. The intrinsic activity for all crystals under test was measured with a HpGe detector. The measured intrinsic activity spectrum is shown in figure 1 . While the intrinsic activity of PrLuAg crystals is not negligible $(\sim 36 \mathrm{~Bq} / \mathrm{g})$, the intrinsic activity of CeCAAG crystals is minimal $\left(\leq 1.5 \times 10^{-3} \mathrm{~Bq} / \mathrm{g}\right)$. The activity of $\mathrm{Ce}: \mathrm{LaBr}_{3}(\sim 0.2 \mathrm{~Bq} / \mathrm{g})$ is half-way between the two.
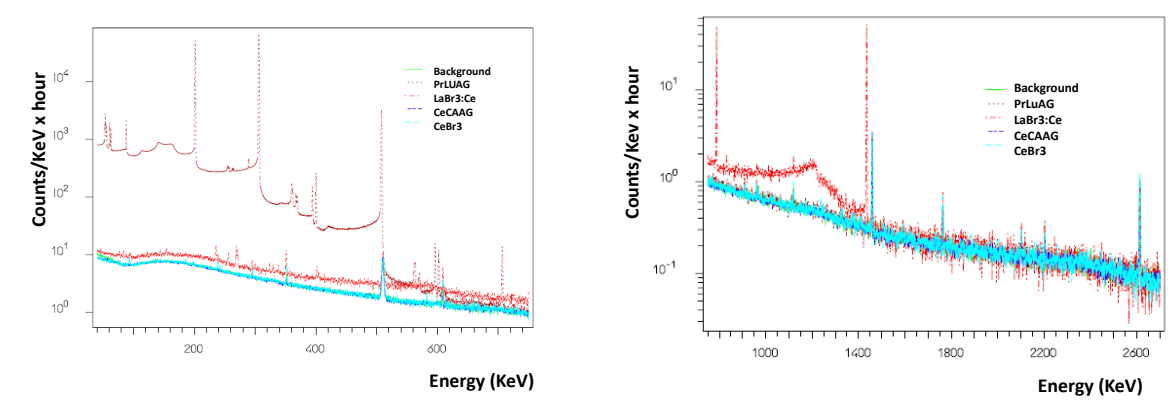

Figure 1: Intrinsic activity of the crystals under test, as measured with a HpGe detector, with a standard spectroscopic chain, based on a Ortec 672 spectroscopy amplifier and a MCA analyzer .

The $1 / 2$ " crystals under test are read by $4 \times 4$ SiPM arrays made of $3 \times 3 \mathrm{~mm}^{2} \mathrm{SiPM}$ from SenSL, Advansid or Hamamatsu. Operating voltages $\left(V_{o p}\right)$ are set according to manufacturer's specs. Their response (PDE) to light of different wavelength depends also on the type of window used, as shown in figure 2 for Hamamatsu S13361 SiPM arrays. Most of our results were obtained

\footnotetext{
${ }^{1} \tau_{1 / 2}=3.78 \cdot 10^{10}$ years, $2.59 \%$ abundance
} 

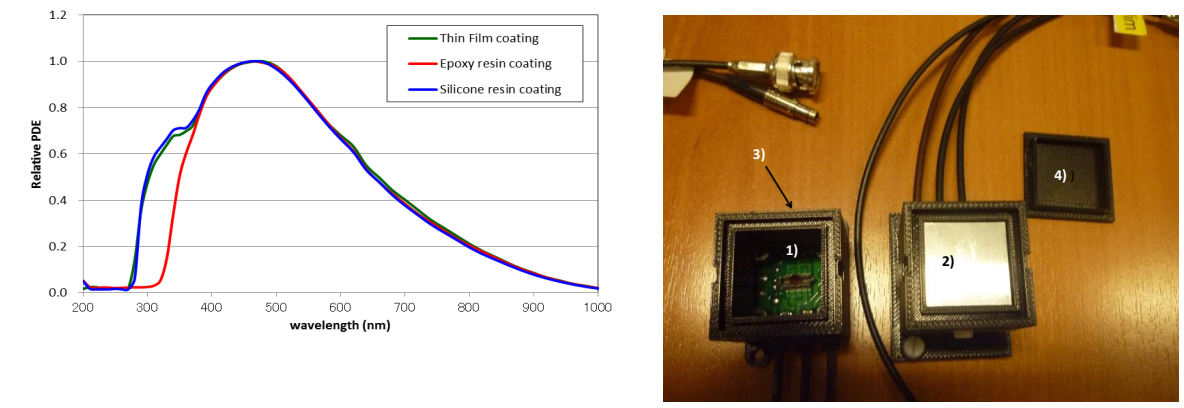

Figure 2: Left panel: response of Hamamatsu S13361 SiPM arrays to incident photons of different wavelengths and with different windows [courtesy of Hamamatsu Italia]. Right panel: crystal mounting. 1) is the PCB; 2) the crystal under test; 3) the crystal holder and 4) the cover to ensure light-tightness.

with Hamamatsu S13361 arrays, based on a TSV ("through silicon via") technology. For PrLuAg (with emission in the near UV) and $\mathrm{LaBr}_{3}$ crystals SiPM arrays with a Silicone window were used to increase response at NUV wavelengths.

The output from each pixel of the $4 \times 4$ SiPM array is summed up on a custom PCB ("basette"). The crystal and the "basette" are mounted inside a light-tight housing printed with a 3D printer (see the right panel of figure 2 for details). The optical coupling between the crystal and the SiPM array was obtained with a Bicron BC630 optical grease ${ }^{2}$. Five out of six faces of the crystal were covered with an optical reflector. As the PrLuAg emission is around $310 \mathrm{~nm}$ it was difficult to find a proper optical diffuser or reflector. The best solution was to use the water based Avian-B optical coating (mainly based on $\mathrm{BaSO}_{4}$ ) that has a $\geq 97 \%(93 \%)$ reflectance in the range 400-850 (250-400) nm. $\mathrm{An} \mathrm{Al}$ diffuser was used instead for CeCAAG crystals.

\section{Experimental results}

Detectors' response has been studied at room temperature, using a standard spectroscopic chain based on various types of amplifiers ${ }^{3}$ and a CAEN N957 MCA. The acquired data, written in ASCII format, were then analyzed with the ROOT package. Temperature excursions in the test setup were monitored with a precision thermometer ${ }^{4}$. The best results were obtained with the Ortec 672 Spectroscopy amplifier, with a $3 \mu$ s shaping time. In the other cases energy resolutions were a 10-30 \% worse. Figure 3 shows a typical MCA spectrum obtained from a PrLuAg $\left(\mathrm{LaBr}_{3}: \mathrm{Ce}\right)$ crystal read by a S13361 Hamamatsu SiPM array, using a $C^{137}$ source.

With a PrLuAg (CeCAAG) crystal the best resolution obtained is around 7\% (6\%). The result obtained for PrLuAg is worse as compared with what obtained with a PMT readout in reference [3], but is better compared with what obtained with a SiPM array readout in reference [6]. Probably this is due to the lower dark noise of the new TSV S13361 Hamamatsu SiPM arrays used. As a reference, with $\mathrm{LaBr}_{3}: \mathrm{Ce}$ crystals we obtain at the $C s^{137}$ peak a resolution better than $4 \%$. Typical results obtained for the crystals under test, with SiPM array readout, are resumed in table 2.

\footnotetext{
${ }^{2}$ with a nearly flat transmission (95\%) between 280 and $700 \mathrm{~nm}$

${ }^{3}$ Ortec 672, Ortec 579, Phillips Scientific PLS774 and CAEN A1423 amplifiers were used

${ }^{4}$ Hanna Checktemp 1 with $0.1^{0} C$ resolution and $\pm 0.3^{0} C$ accuracy
} 

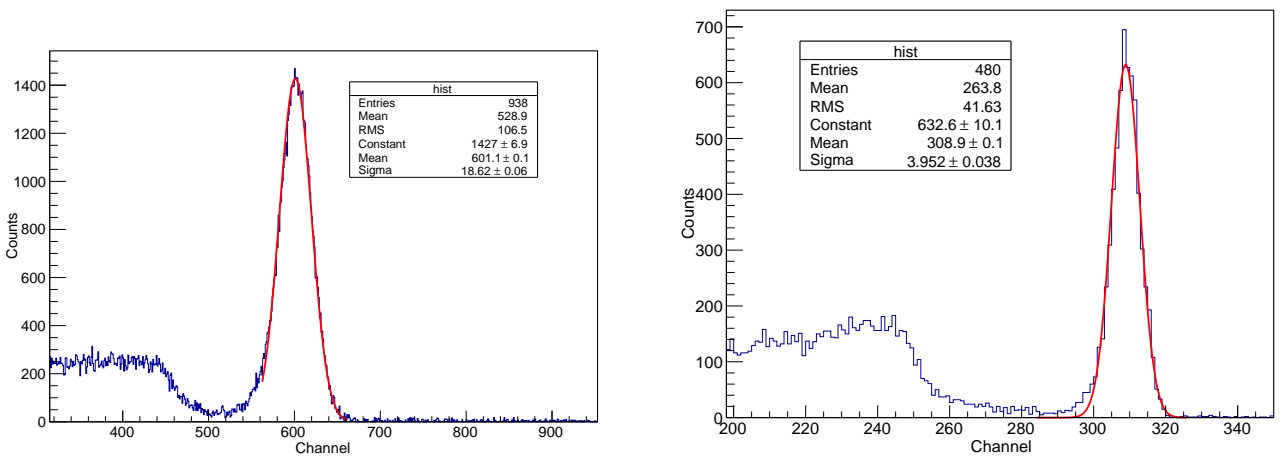

Figure 3: Energy spectrum of the $662 \mathrm{keV}$ gamma rays, as measured with the $14 \times 14 \times 13 \mathrm{~mm}^{3} \mathrm{PrLuAg}$ (left) and $\mathrm{LaBr}_{3}$ (right) crystals coupled to the MPPC array (Hamamatsu S13361 with a Silicone window). MCA counts are reported on the abscissa. Plots are not corrected for linearity.

Table 2: Obtained FWHM energy resolutions (in \%) at the $\mathrm{Cs}^{137}$ peak for some specimen of the crystals under test. H/A refers to the used Hamamatsu S13361/Advansid ASD-P-4-TD NUV SiPM arrays.

\begin{tabular}{|l|c|c|c|c|c|c|}
\hline resolution & $\mathrm{LaBr}_{3}(\mathrm{H})$ & $\mathrm{LaBr}_{3}(\mathrm{~A})$ & $\mathrm{CeBr}_{3}(\mathrm{H})$ & CeCAAG $(\mathrm{H})$ & $\operatorname{PrLuAg}(\mathrm{H})$ & $\mathrm{NaI}(\mathrm{Tl})(\mathrm{H})$ \\
\hline Ortec 672 & $3.1 \%$ & $3.8 \%$ & $4.7 \%$ & $6.4 \%$ & $7.2 \%$ & $8.4 \%$ \\
Ortec 579 & $3.4 \%$ & $4.3 \%$ & $5.0 \%$ & - & - & $8.9 \%$ \\
\hline
\end{tabular}

As LaBr3:Ce crystals with a SiPM array readout give a sizeable signal in the $100-200 \mathrm{mV}$ range, a simple FADC readout scheme may be implemented. The signal is either shaped by an Ortec 672 spectroscopic amplifier and then fed to a CAEN V1730 FADC( 12 bit, $500 \mathrm{Ms} / \mathrm{s}$ ) or directly fanned into the FADC. Bias to the SiPM is common for all the 16 cells of an array and is given by an ISEG NHS 6001 NIM module.
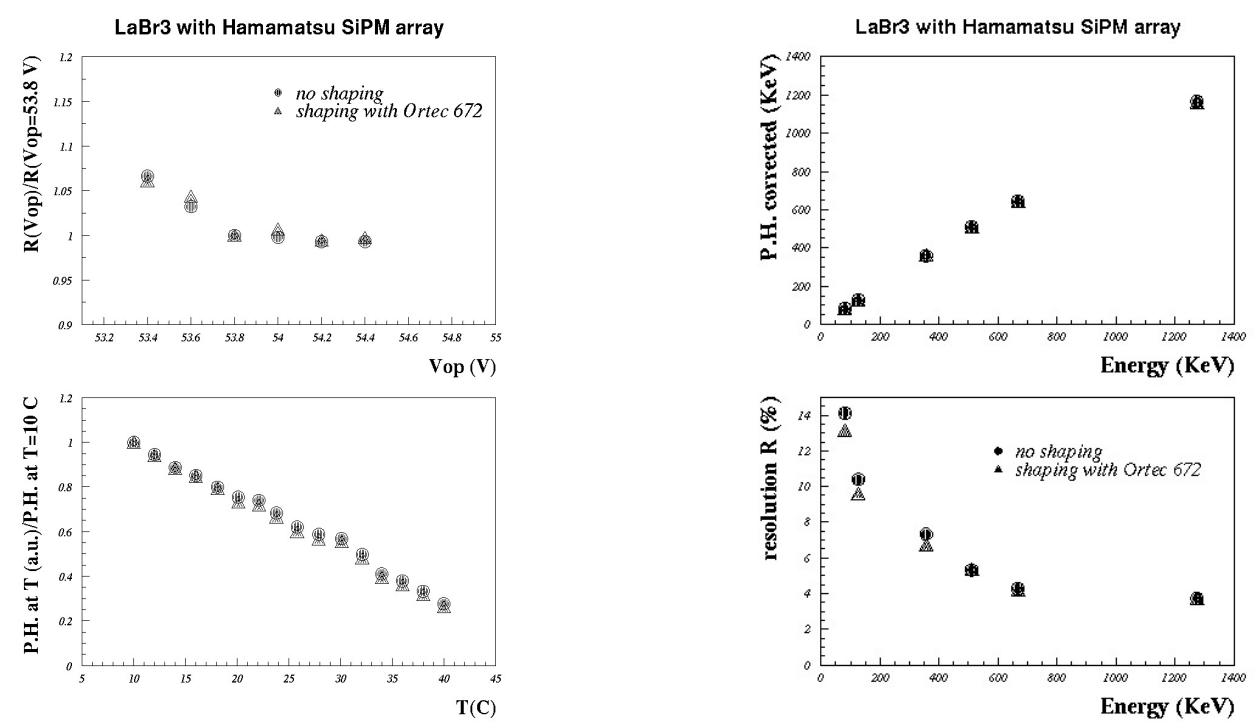

Figure 4: Left top panel: energy resolution (FWHM) dependence for $\mathrm{LaBr}_{3}$ :Ce crystals from the operating voltage $\left(V_{o p}\right)$. Left bottom panel: dependence of the detector response from temperature, at fixed $\mathrm{V}_{o p}=53.8$ $\mathrm{V}$. Right panel: linearity and energy resolution of a typical $\mathrm{LaBr}_{3}$ crystal, at room temperature .

The variation of energy resolution at the $\mathrm{Cs}^{137}$ peak for $\mathrm{LaBr}_{3}$ crystals with Hamamatsu readout, as a function of the operating voltage $V_{o p}$, is shown in the left panel of figure 4 together with 
the dependence of the response from temperature, measured inside a Memmert IPV30 climatic chamber. The right panel of figure 4 shows instead the linearity and the resolution (FWHM) for a typical $\mathrm{LaBr}_{3}$ crystal under test at $V_{o p}=53.8 \mathrm{~V}$ using $\mathrm{Na}^{22}, \mathrm{Cs}^{137}, \mathrm{Ba}^{133}$ and $\mathrm{Co}^{57}$ exempt sources.

For effective use in experimental conditions, one has to take into account the SiPM gain drift with temperature. In the present setup, a thermistor is mounted on the "basette", to make an offline correction afterwards. In the near future, we plan to correct online the SiPM gain drift by using a NIPM-12 SiPM power module from Nuclear Instruments srl Lambrugo (IT) that integrates a temperature HV loop, to regulate the SiPM bias voltage as a programmable function of the SiPM temperature coefficient.

\section{Conclusions}

Preliminary results for the used crystals with a SiPM array readout show that they are promising for what regards energy resolution, even if performances for PrLuAg are not yet optimal. Their possible use with low-energy $\mathrm{X}$ rays needs further optimization. Good results are obtained with more conventional $\mathrm{LaBr}_{3}$ :Ce crystals using the new low-noise NUV SiPM arrays from Hamamatsu and Advansid, making this our best choice for the time being. A simple electronics front-end based on CAEN V1730 FADC, without an amplification stage, gives resolution (FWHM) at the $C s^{137}$ peak better than $4 \%$, comparing well with the best available results.

\section{Acknowledgements}

We acknowledge the help of S.Banfi and F. Chignoli (INFN Milano Bicocca) for mechanics and electronics setup mounting. We would like to thank also Dr. E. Vallazza (INFN Trieste) for his friendly help in implementing the data acquisition for the test setup.

\section{References}

[1] K. Kamada et al., Basic experiments on radiation imaging by using $\mathrm{Pr}: \mathrm{Lu}_{3} \mathrm{Al}_{5} \mathrm{O}_{12}(\mathrm{LuAG}$ ) small crystalline pixels with various reflectors, IEEE Trans. Nucl. Science N24-208 (2007) 1417.

[2] A. Vacchi et al., Measuring the size of the proton, SPIE Newsroom (2012); doi: 10.1117/2.1201208.004274;

A. Adamczak et al., Steps towards the hyperfine splitting measurement of the muonic hydrogen ground state: pulsed muon beam and detector system characterization, JINST 11/5 P05007 (2016); doi: 10.1088/1748-0221/11/05/P05007.

[3] W. Drozdowski et al., Scintillation Properties of Praseodymium Activated $\mathrm{Lu}_{3} \mathrm{Al}_{5} \mathrm{O}_{12}$ Single Crystals, IEEE Trans. Nucl. Science 55 (2008) 2429

[4] J.Y. Yeom et al., First Performance Results of Ce:GAGG Scintillation Crystals With Silicon Photomultipliers, IEEE Trans Nucl Science 60, no.2 (2013) 988.

[5] F.G.A Quarati et al., Scintillation and detection characteristics of high-sensitivity CeBr $r_{3}$ gamma-ray spectrometers, Nucl. Instr. Meth. A729 (2012) 596; doi: 10.1016/j.nima.2013.08.005.

[6] T. Kato et al., Development of a large-area monolithic 4x4 MPPC array for a future PET scanner employing pixelized Ce:LYSO and Pr:LuAg crystals, Nucl. Instr. Meth. A638 (2011) 83; doi: 10.1016/j.nima.2011.02.049. 